\title{
The benefit of being good
}

The problem of altruism - understanding how natural selection creates individuals who help others at their own expense - is among the most basic puzzles of biology. Why, in a world of cut-throat competition, aren't such organisms driven out of existence? As a fundamental puzzle of mathematical logic, this issue holds a natural interest for many physicists. Given what we know about the dynamics of evolution, the problem is simply to specify the conditions, if they exist, under which we should expect altruism to flourish.

But the simplicity of the question is not matched by the answers currently known, which point to a bewildering assortment of mechanisms able to produce altruism. A pair of selfish organisms, for example, can readily learn through repeated encounters that each does better by cooperating: this is 'reciprocal altruism. The theory of 'kin selection' explains why brothers and sisters and other close genetic relatives cooperate, as these individuals thereby further their own genes, many copies of which reside in their close kin. These are only two of perhaps dozens of scenarios so far identified.

I've often wondered if there isn't a confusing surplus of explanations and a lack of any unifying theme. Hence my excitement over an effort by biologists Jeffrey Fletcher and Michael Doebeli to elucidate such a theme (Proc. R. Soc. B 276, 13-19; 2009). They assert that the real key to altruism is simply the existence of some mechanism and there are likely to be many - that biases the interactions between individuals so that altruists tend to meet up preferentially with others who help them. In short, everything depends on the non-randomness of interactions between individuals of different types.

Their argument starts with the observation that for any genotype to increase its frequency in a population, individuals carrying it must, on average, receive greater net benefit than others in the population. Altruists sometimes act in ways that disadvantage themselves relative to others they may even sacrifice themselves so that others may live - but individuals of this type must on average receive more than enough positive returns to compensate.

Fletcher and Doebeli argue that this point holds the key to the puzzle, if it is considered properly. Imagine a population of two types of individuals, cooperators or defectors, engaged in a so-called public goods game, which you might think of as the 'hydrogen

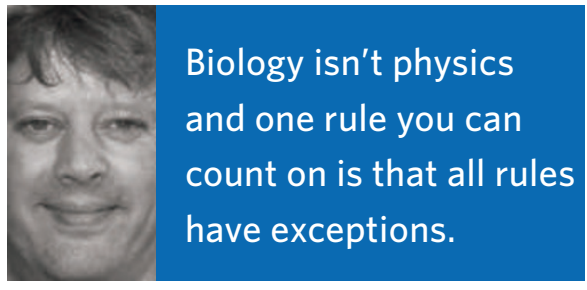

atom' of altruism. Individuals meet and interact in a group of size $N$. If they choose, each person can contribute a value $b$ to the public good - a fund benefiting all and after the game, each then collects an amount from this fund in proportion to how many contributed. (Think of taxes spent on better roads, education, and so on - goods that benefit all.)

Cooperators and defectors behave differently. Cooperators always make the contribution; doing so costs them an amount $c$. In contrast, defectors are like tax cheats; they never contribute to the public good, and so pay no costs. Still, like cooperators, they will happily benefit from the fund. So what happens?

In this simple game, if $k$ individuals were cooperators, and $N-k$ defectors, then the total good produced is $k b$. This amount is shared out equally among all $-k b / N$ to each. So a cooperator gets a total of $k b / N-c$, whereas a defector gets $k b / N$. The altruists always lose out, and this is true no matter what the value of $k$ (supposing that $k<N$, meaning that not everyone cooperates). Altruism doesn't work in this setting.

Fletcher and Doebeli next consider a more general problem: the same publicgoods game, but now in a large population. Individuals still meet and interact in groups of size $N$, but the number of cooperators and defectors fluctuates from one group to the next. It's the average character of these groups that now matters most. If a cooperator encounters on average $e_{\mathrm{C}}$ other cooperators, while defectors encounter on average $e_{\mathrm{D}}$ other cooperators, then the average pay off for a cooperator is $e_{\mathrm{C}} b / N$ (coming from others) $+b / N-c$ (the part coming from the cooperator's action itself); for defectors it is $e_{\mathrm{D}} b / N$ (coming only from others' actions).

If individuals enter these groups completely at random, then in this case, $e_{\mathrm{C}}=e_{\mathrm{D}}-$ cooperators and defectors see the same environment of others - and we find that altruism requires the condition $b / N-c>0$. This is the result in the context of total randomness in associations, and hence a useful baseline. If the act really is altruistic - that is, the share coming to a cooperator from their own contribution isn't enough to outweigh the cost $(b / N-c<0)-$ then an altruist loses out. In a random population, true altruism cannot exist, only a 'weak' variety in which the altruist's action benefits itself immediately, which isn't really what we mean by altruism.

But this only sets up the most interesting point. As the authors explain, everything changes in the non-random case in which $e_{\mathrm{C}} \neq e_{\mathrm{D}}$. Suppose that something structures the interactions between individuals, making some individual types more likely to interact than others. It may make some cooperators, for example, tend to meet more readily with other cooperators. It could then be that $e_{\mathrm{C}}>e_{\mathrm{D}}$, and a little algebra then gives the condition for altruism as $e_{\mathrm{C}}-e_{\mathrm{D}}>c N / b-1$. Now, even when $b / N-c<0-$ for which an altruist's actions really are costly to itself - altruism can still emerge by virtue of the good that comes back to an altruist from others.

Fletcher and Doebeli go on to examine how each of the known theories for altruism illustrates this picture in some specific circumstance. Each theory emphasizes different mechanisms that cause an assortment of interactions, such as the limited movement of a population (sometimes called population viscosity, which produces geographical restrictions on who interacts), kin recognition, group structures and so on. Hence, they argue, these seemingly different fundamental explanations are really the same, only in superficially different clothing.

This to me seems to be an important achievement. But there is more. The general rule stated by Fletcher and Doebeli is that altruists must meet up preferentially with others who help them. 'Others', as they point out, needn't always be other altruists of the same kind. For example, it might well involve interactions between different species.

Whether Fletcher and Doebeli's discussion really unifies this fascinating but muddled area of science remains to be seen. Biology isn't physics and it seems that one rule you can count on is that all rules have exceptions. But there's always a place for efforts of this kind - editing, as it were, existing scientific knowledge, seeking simplicity and unity.

MARK BUCHANAN 